\title{
Optimization study on the rehydration process of lyophilized human platelets
}

\author{
FAN JuLi ${ }^{1,2}$, XU XianGuo ${ }^{3,4}$, ZHANG ShaoZhi $^{2 *}$, XU MengJie ${ }^{2}$, ZHU FaMing $^{3,4}$ \& \\ CHEN GuangMing ${ }^{2}$ \\ ${ }^{1}$ Department of Man-machine and Environment Engineering, Nanjing University of Aeronautics and Astronautics, Nanjing 210016, China; \\ ${ }^{2}$ Institute of Refrigeration and Cryogenics, Zhejiang University, Hangzhou 310027, China; \\ ${ }^{3}$ Blood Center of Zhejiang Province, Hangzhou 310006, China; \\ ${ }^{4}$ Key Laboratory of Blood Safety Research, Ministry of Health, Hangzhou 310006, China
}

Received October 31, 2009; accepted February 28, 2010

\begin{abstract}
Long-term preservation of human platelets will greatly reduce the risk of their shortage. Lyophilization has been proved feasible for this purpose. For the recovery of lyophilized platelets, rehydration is an important process. In this paper, the rehydration processes for $1 \mathrm{~mL}$ and $2 \mathrm{~mL}$ samples were studied. The effects of prehydration duration $(15,30,60,90,120$ and $150 \mathrm{~min})$ in $37^{\circ} \mathrm{C}$ water vapor and the concentration of rehydration solution $(25 \%, 50 \%, 75 \%, 100 \%$ platelet-poor plasma) on the recovery rate, MPV (mean platelet volume) and PDW (platelet distribution width) were investigated. The mass changes during the prehydration process were weighed. The optimized rehydration conditions are as follows: (1) for $1 \mathrm{~mL}$ sample, the prehydration duration was $15 \mathrm{~min}$ and for $2 \mathrm{~mL}$ sample the prehydration duration was $90 \mathrm{~min}$; (2) the rehydration solution was 75\% platelet-poor plasma. Under optimized conditions, the morphology of the rehydrated platelets kept normal and their ultrastructures kept intact, their aggregation capacity to thrombin $(1 \mathrm{U} / \mathrm{mL})$ was $82.8 \%$ of the fresh ones. These results will be helpful for designing the freeze-drying protocols for human platelets.
\end{abstract}

lyophilization, human platelets, prehydration, rehydration solution, morphology, aggregation function

Citation: Fan J L, Xu X G, Zhang S Z, et al. Optimization study on the rehydration process of lyophilized human platelets. Chinese Sci Bull, 2011, 56: 455-460, doi: $10.1007 / \mathrm{s} 11434-011-4381-7$

Human platelets are very useful in clinical treatments, such as enhancing wound healing [1-3]. Usually, in blood banks, platelets are stored at room temperature, $22^{\circ} \mathrm{C}$, which has limited shelf life of several days. Cryopreservation provides an alternative for the long-term storage of platelets, but the washing of cryoprotectant prior to use is bothersome and may bring damages to the cells [4]. The expensive equipments such as low temperature refrigerator and special $\mathrm{LN}_{2}$ tank also make cryopreservation not feasible for bulk storage. Since the supply and the demand of human platelets are fluctuating seasonally, it is of great importance to establish a method that can store platelets conveniently and cheaply.

*Corresponding author (email: enezsz@ zju.edu.cn)
Lyophilization has been widely used in the delivery of blood products and vaccines [5-8]. If human platelets have been successfully freeze-dried, they would have several advantages over the traditional methods [9]: (1) lyophilized platelets could be stored at room temperature for several months without any cryo-equipments; (2) lyophilized platelets could be reconstituted quickly at the point of use, which is especially important in emergency; (3) lyophilized platelets could be shipped and transported readily. In 1995, Read et al. lyophilized the paraformaldehyde-fixed platelets, but the recovery of those platelets was low [10]. As the research in cryobiology deepens, the lyophilization of human platelets finally has a breakthrough. In 2001, Wolkers et al. freeze-dried platelets and made the recovery rate of $85 \%$, 
with the protection of trehalose [11]. And they developed a freeze-drying method of platelets of large volume [12,13]. In 2007, the authors' research group optimized the freezedrying protocol of human platelets with respect to lyoprotectant, cooling rate and cell concentration [14]. There remain a lot of subjects to be studied in the freeze-drying of mammalian cells, such as the rehydration process. Wolkers et al. found prehydration had good effects on the morphology of rehydrated freeze-dried human platelets [11]. However, they did not carry out the in depth study for this issue. Han et al. tested different rehydration solutions at different temperatures, and found that the survival rate of rehydrated erythrocytes was more dependent on colloidal osmotic pressure than on crystalloid osmotic pressure [15]. Shu et al. studied the effects of the temperature $\left(0,10,25,37,42^{\circ} \mathrm{C}\right)$ of the rehydration solution of lyophilized red blood cells and got the highest cell recovery rate at $37^{\circ} \mathrm{C}$ [16]. Fan et al. studied the rehydration process of $1 \mathrm{~mL}$ lyophilized platelets and obtained the following findings: (1) prehydration is significantly beneficial for the recovery of platelets; (2) there is no obvious difference for two prehydration temperatures, $35^{\circ} \mathrm{C}$ and $37^{\circ} \mathrm{C}$; (3) $50 \%$ platelet-poor plasma behaves better than PBS as rehydration solution [17].

In this paper, the rehydration process of the freeze-dried platelets will be further studied. The prehydration duration and the concentration of the rehydration solution (plateletpoor plasma) will be optimized. The effect of the sample volume or thickness will also be considered. Similar to the previous study, the recovery rate, MPV, and PDW of the rehydrated platelets will be used as viability standards. The water contents of the samples at several time points of the prehydration process will also be recorded. Moreover, the morphology and the aggregate function of the freeze-dried platelets rehydrated with the optimized protocol will be investigated.

\section{Materials and methods}

(i) Platelets preparation. The preparation of platelets was the same as Fan et al. [17]. Platelets samples were incubated to load trehalose and then suspended in the lyoprotectants. The lyoprotectants include $528.6 \mathrm{mmol} / \mathrm{L}$ trehalose and $1 \%$ $(\mathrm{w} / \mathrm{v})$ bovine serum albumin (BSA). The cell count, the mean platelet volume (MPV) and the platelet distribution width (PDW) were measured by a CELL-DYN1700 hemacytometer (Abbott, USA). The cell concentration in the lyophilization buffer was about $1 \times 10^{9} / \mathrm{mL}$.

(ii) Freezing. Aliquots of $1 \mathrm{~mL}$ and $2 \mathrm{~mL}$ platelet suspensions were filled into glass vials (diameter, $25 \mathrm{~mm}$ ) and frozen at $-60^{\circ} \mathrm{C}$ for $2 \mathrm{~h}$, with a cooling rate of about $10^{\circ} \mathrm{C}$ $/ \mathrm{min}$.

(iii) Freeze-drying. The freeze-drying was performed in a laboratory-scale freeze-drier (Labconco, USA) with the following four steps: (1) the freeze-drier cabinet was precooled to $-40^{\circ} \mathrm{C}$ at a rate of $1.5^{\circ} \mathrm{C} / \mathrm{min}$ while the cold con- denser was cooled to $-80^{\circ} \mathrm{C}$; (2) the frozen samples were put onto the shelf of freeze-drier cabinet, and the vacuum pump started to work; (3) for $1 \mathrm{~mL}$ samples primary drying, it lasted for $16 \mathrm{~h}$ and for $2 \mathrm{~mL}$ samples primary drying, it lasted for $35 \mathrm{~h}$; (4) the shelf was warmed up to $22^{\circ} \mathrm{C}$ at a rate of $0.2^{\circ} \mathrm{C} / \mathrm{min}$ and then kept at that temperature for $16 \mathrm{~h}$ for $1 \mathrm{~mL}$ samples, for $2 \mathrm{~mL}$ samples the time was $20 \mathrm{~h}$.

During the drying process, the vacuum chamber pressure was kept at about $1 \mathrm{~Pa}$. After freeze-drying, the samples were sealed with rubber stoppers automatically and stored at room temperature.

(iv) Rehydration. Some samples of lyophilized platelets were prehydrated in a vessel with saturated air at $37^{\circ} \mathrm{C}$ (equivalent to a partial pressure of water vapor of $6.3 \mathrm{kPa}$ ) for $15,30,60,90,120$ or $150 \mathrm{~min}$, respectively. After prehydration, each sample was rehydrated in the platelet-poor plasma solution with the concentration of $50 \%$ at room temperature of $22^{\circ} \mathrm{C}$, followed by gentle shaking until the sample was completely dissolved in the rehydration solution. Some $1 \mathrm{~mL}$ samples were directly rehydrated in $1 \mathrm{~mL}$ platelet-poor plasma solutions with the concentrations of $25 \%$, $50 \%, 75 \%$ or $100 \%$, at $22^{\circ} \mathrm{C}$.

(v) Haematology analysis. The numerical recovery of rehydrated platelets was determined by counting platelets with a CELL-DYN1700 hemacytometer (Abbott, USA). The mean platelet volume (MPV) and the platelet distribution width (PDW) after rehydration were measured and recorded by the same hemacytometer.

$$
\begin{aligned}
& \text { Numerical recovery of lyophilized platelets } \\
& =\frac{\text { the number of platelets after freeze }- \text { drying and rehydration }}{\text { the number of platelets before freeze }- \text { drying }}
\end{aligned}
$$

(vi) Water content measurement. The water content of the samples was determined with the help of an electronic analytical balance (Mettler AE200, $0.0001 \mathrm{~g}$, Switzerland) and a thermogravimetry (TA Instruments Q600, USA).

The water contents $\left(C_{w}, \mathrm{~g}\right.$ water/g wet basis) of prehydrated lyophilized platelets were calculated as follows:

$$
C_{w}=\left[\left(W_{d}-W_{e}\right) \times C_{d}+\left(W_{p}-W_{d}\right)\right] \times 100 \% /\left(W_{p}-W_{e}\right),
$$

where $W_{e}$ is the weight of the empty vial, $W_{d}$ is the weight of the vial with lyophilized platelets, $W_{p}$ is the weight of the vial with prehydrated samples, $C_{d}$ is the water content of lyophilized platelets.

(vii) Scanning electron microscopy /transmission electron microscopy(SEM/TEM). Rehydrated platelets were observed with both scanning and transmission electron microscopy. The samples for SEM were made as follows: (1) the samples were fixed with $2.5 \%$ glutaraldehyde at $4{ }^{\circ} \mathrm{C}$; (2) $0.1 \mathrm{~mol} / \mathrm{L}$ PBS was used to rinse the samples twice for 15 min; (3) the samples were fixed with $1 \%$ osmic acid for $1 \mathrm{~h}$; (4) the samples were dehydrated by increasing concentrations of ethanol $(50 \%, 70 \%, 80 \%, 90 \%$ for $15 \mathrm{~min}$, and $100 \%$ twice for $15 \mathrm{~min}$ ); (5) the samples were dehydrated through $100 \%$ acetone for $15 \mathrm{~min}$ and $100 \%$ isoamyl acetate 
for $15 \mathrm{~min}$; (6) the samples were dried using an HCP-2 critical-point-drier (Hitachi, Japan); (7) the samples were sputtered with gold in an E-1020 ion sputter (Hitachi, Japan). The prepared samples were analyzed with Stereoscan-260 SEM (Cambridge Instrument, UK) at $20 \mathrm{kV}$.

The samples for TEM were made as follows: Steps (1-3) were the same as the above processes for SEM; (4) Step 2 was repeated; (5) the samples were stained with $2 \%$ uranyl acetate for $30 \mathrm{~min}$; (6) the samples were dehydrated by increasing the concentrations of ethanol $(50 \%, 70 \%, 90 \%$ for $15 \mathrm{~min}$, and $100 \%$ for $20 \mathrm{~min}$; (7) the samples were dehydrated through $100 \%$ acetone twice for $20 \mathrm{~min}$; (8) the samples were embedded and collected at $37^{\circ} \mathrm{C}$ for $24 \mathrm{~h}, 45^{\circ} \mathrm{C}$ for $24 \mathrm{~h}, 60^{\circ} \mathrm{C}$ for $48 \mathrm{~h}$; (9) the samples were cut as thin slices; (10) the slices were stained with $2 \%$ uranyl acetate for $20 \mathrm{~min}$, with lead citrate for $15 \mathrm{~min}$; (11) the prepared samples were analyzed with Tecnai-10 TEM (Philips, Holand).

(viii) Aggregation test. The aggregation function of rehydrated platelets were estimated and compared with fresh platelets. Each sample was filled into an aggregation cuvette with a stirring bar inside and kept at $37^{\circ} \mathrm{C}$. Then, thrombin was added into the platelet suspension in turns to a final thrombin concentration of $1 \mathrm{U} / \mathrm{mL}$. The aggregation traces of the platelets were recorded with an aggregometer (Chrono-Log, USA).

(ix) Statistical analysis. The experimental data were statistically analyzed using one-way ANOVA. A level of $P \leqslant 0.05$ was accepted as being statistically significant.

\section{Results}

\subsection{Haematology results}

Table 1 shows the recovery rates of the experiments on prehydration duration with the sample volumes of $1 \mathrm{~mL}$ and $2 \mathrm{~mL}$. For $1 \mathrm{~mL}$ platelets samples, as prehydration duration increases from $15 \mathrm{~min}$ to $120 \mathrm{~min}$, the platelets recovery decreases from $89 \%$ to $77 \%$. The difference between $15 \mathrm{~min}$ group and 60 min group is statistically significant. For $2 \mathrm{~mL}$ samples, prehydration for $90 \mathrm{~min}$ leads to the highest platelets recovery, and the difference between $90 \mathrm{~min}$ group and 60 min group is statistically significant.

Table 1 Platelets recovery rehydrated with $50 \%$ plasma $(n=4)$

\begin{tabular}{cll}
\hline \multirow{2}{*}{ Prehydration duration $(\min )$} & \multicolumn{2}{c}{ Platelets recovery $(\%)$} \\
\cline { 2 - 3 } & $1 \mathrm{~mL}$ & \multicolumn{1}{c}{$2 \mathrm{~mL}$} \\
\hline 0 & $80 \pm 7.4$ & $68 \pm 5.2$ \\
15 & $89 \pm 9.4^{\mathrm{a})}$ & $70 \pm 4.7$ \\
30 & $86 \pm 8.4$ & $72 \pm 3.3$ \\
60 & $79 \pm 6.9^{\mathrm{a})}$ & $68 \pm 4.6^{\text {b) }}$ \\
90 & $78 \pm 7.0$ & $85 \pm 7.5^{\mathrm{b}), \mathrm{c})}$ \\
120 & $77 \pm 5.3$ & $80 \pm 3.4^{\mathrm{c}}$ \\
\hline
\end{tabular}

a) $P=0.03<0.05$, b) $P=0.01<0.05$, c) $P=0.29>0.05$.
Figure 1(a) and (b) show the changes of MPV and PDW values for various prehydration durations. It can be seen that for $1 \mathrm{~mL}$ samples, the platelets prehydrated for $15 \mathrm{~min}$ have the smallest average MPV and the lowest average PDW, close to the MPV for fresh human platelets, $11.81 \% \pm 0.90 \%$ $\mathrm{fL}$ and the PDW for fresh human platelets $17.02 \% \pm 1.10 \%$ [18]. Further ANOVA analysis demonstrates that significant MPV and PDW differences exist between 15 min group and other groups. Therefore, $15 \mathrm{~min}$ or less may be the most appropriate prehydration duration for $1 \mathrm{~mL}$ freeze-dried human platelet samples. And for $2 \mathrm{~mL}$ samples, prehydration for 60 or 90 min has the best MPV, and prehydration for $90 \mathrm{~min}$ has the best PDW. Therefore, $90 \mathrm{~min}$ may be the most appropriate prehydration duration for $2 \mathrm{~mL}$ freezedried human platelet samples.

Figure 2 shows the whole PDW curves of $1 \mathrm{~mL}$ samples for fresh and freeze-dried platelets. It can be seen that the PDW curve corresponding to prehydration for $15 \mathrm{~min}$ (Figure 2(c)) is the closest to the PDW curve of the fresh platelet (Figure 2(a)) among the three curves for freeze-dried platelets.

Table 2 shows the platelets recovery rates rehydrated with different concentrations of platelet-poor plasma. As a result, the recovery rate is the highest for the samples rehydrated with $75 \%$ plasma. It is statistically different from the samples rehydrated with $50 \%$ plasma.

Figure 3 shows the PDW curves of rehydrated platelets with different concentrations of plasma. It can be observed that the PDW curve for the samples rehydrated with $75 \%$ plasma (Figure 3(c)) is the closest to PDW curve for fresh platelets among the four curves (Figure 2(a)).

\subsection{Changes of water content}

The water content of freeze-dried samples, $C_{d}$, is about $2.5 \%$. Figure 4 shows the water content $\left(C_{w}\right)$ after prehydration. It can be seen that it increases with the prehydration time, and the water increment for $2 \mathrm{~mL}$ samples is about twice for that of $1 \mathrm{~mL}$ samples. For $1 \mathrm{~mL}$ platelet samples, after prehydration for $15 \mathrm{~min}$, the average water content is $5.3 \% \pm 0.29 \%$, while for $2 \mathrm{~mL}$ samples, after prehydration for $90 \mathrm{~min}$, the average water content is $6.1 \% \pm 0.24 \%$.

\subsection{Morphology of rehydrated lyophilized platelets}

To check the integrity of the ultrastructure morphology of rehydrated platelets with the optimized rehydration process, $1 \mathrm{~mL}$ lyophilized samples were preserved for 30 days, prehydrated for $15 \mathrm{~min}$ or $0 \mathrm{~min}$, and rehydrated with $1 \mathrm{~mL}$ $75 \%$ plasma solution. The recovered platelets were observed with SEM/TEM and compared with the fresh ones.

Figure 5(a) and 5(b) show the SEM photographs of rehydrated platelets with prehydration for $15 \mathrm{~min}$ and $0 \mathrm{~min}$ respectively. It can be seen that the morphology of platelets in Figure 5(a) is more uniform than that in Figure 5(b). 

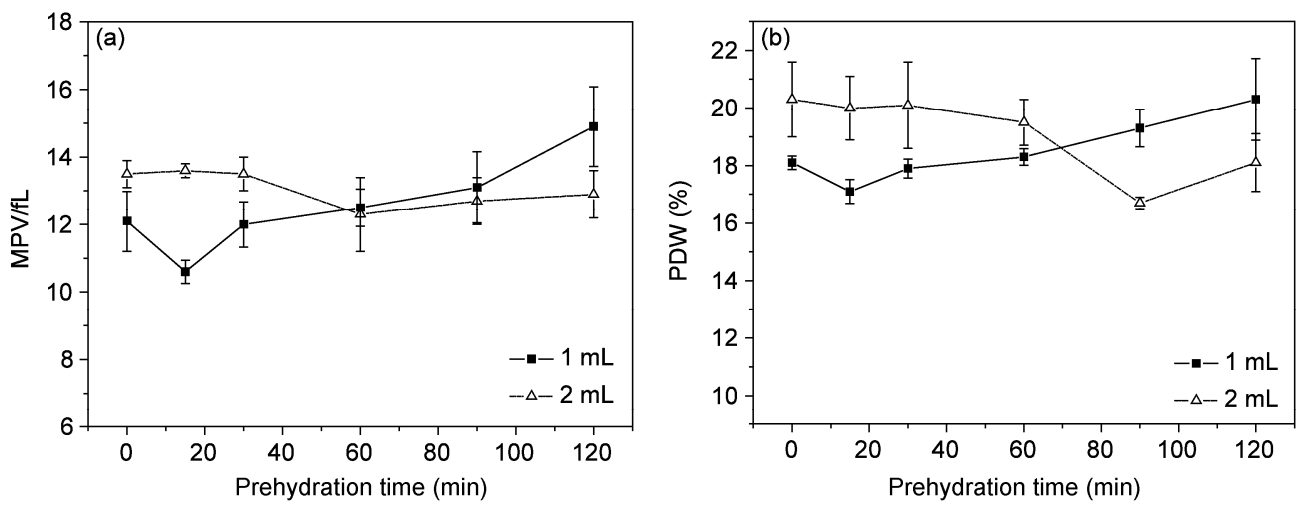

Figure 1 The changes of MPV and PDW with different prehydration durations $(n=4)$. (a) MPV; (b) PDW.
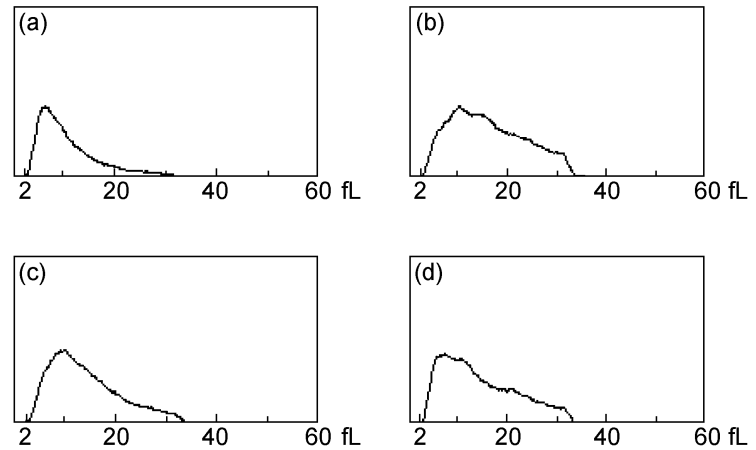

Figure 2 PDW curves of the platelets with different ways of rehydration $(1 \mathrm{~mL})$. (a) Fresh; (b) freeze-dried (directly rehydrated); (c) prehydrated for $15 \mathrm{~min}$; (d) prehydrated for $90 \mathrm{~min}$.

Table 2 Platelets recovery rehydrated with different concentrations of plasma $(n=3)$

\begin{tabular}{cc}
\hline Rehydration solution & Platelets recovery $(\%)$ \\
\hline $25 \%$ plasma & $68 \pm 2.5$ \\
$50 \%$ plasma & $79 \pm 3.4^{\text {a) }}$ \\
$75 \%$ plasma & $89 \pm 3.6^{\text {a),b }}$ \\
$100 \%$ plasma & $83 \pm 2.6^{\text {b) }}$ \\
\hline
\end{tabular}

a) $P=0.03<0.05$, b) $P=0.09>0.05$.
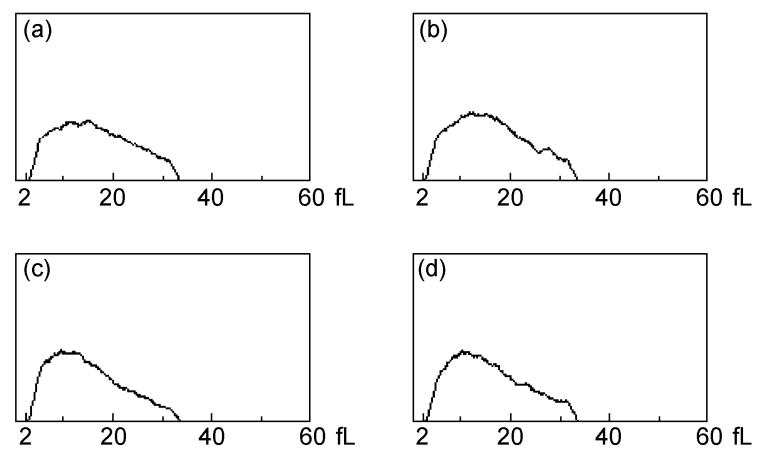

Figure 3 PDW curves of rehydrated platelets with different concentrations of plasma (1 mL). (a) 25\%; (b) 50\%; (c) 75\%; (d) $100 \%$

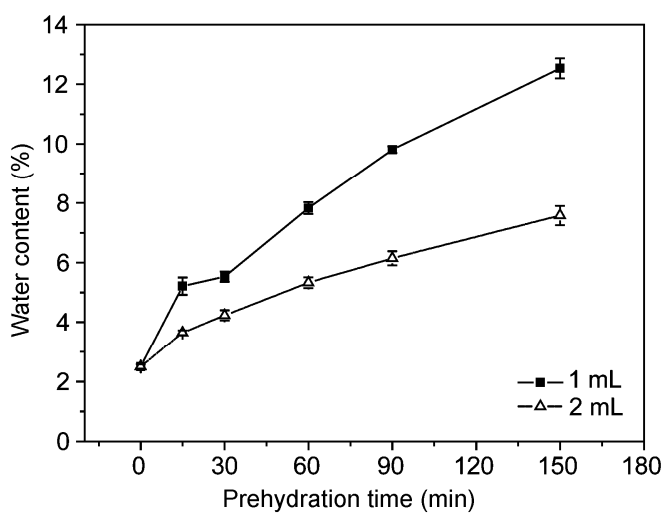

Figure 4 Water content of prehydrated samples $(n=4)$.
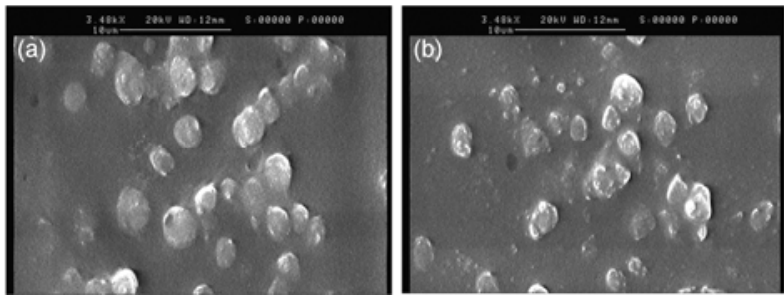

Figure 5 SEM pictures of rehydrated platelets. (a) Optimized rehydrated platelets (3480x); (b) directly rehydrated platelets (3480x).

Moreover, some cell debris can be viewed in Figure 5(b).

Figure 6(a), (b) and (c) show the TEM photographs of fresh and rehydrated platelets. Figure 6(c) shows the optimized rehydrated freeze-dried platelet, Figure 6(b) shows the directly rehydrated freeze-dried platelet, while Figure 6(a) shows the fresh platelet as the control. It can be seen that the ultrastructure of lyophilized platelet with optimized rehydration is integral. It is closer to the ultrastructure of fresh platelet than that of lyophilized platelets with direct rehydration.

\subsection{Response of freeze-dried platelets to thrombin}

The response of fresh and rehydrated freeze-dried platelets to thrombin was investigated by aggregometry. The aggregation traces were shown in Figure 7(a)-(c). The maximum 


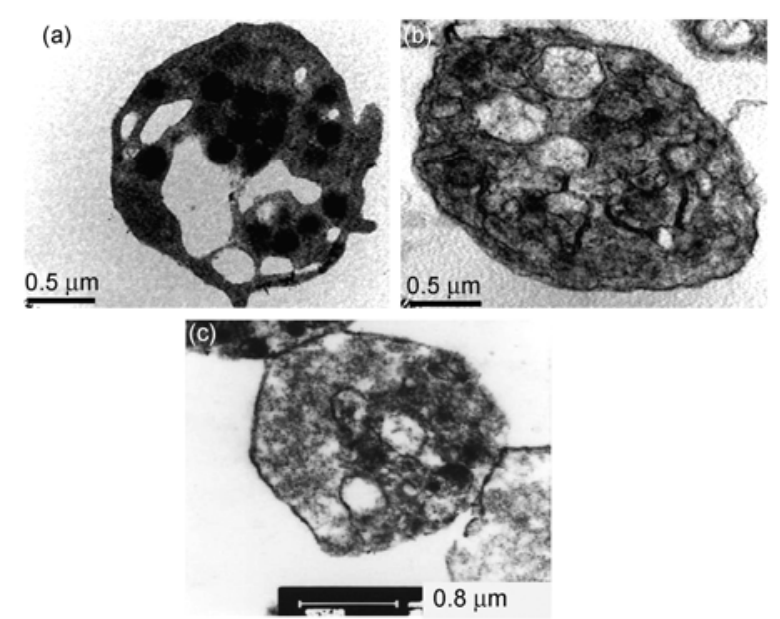

Figure 6 TEM pictures of fresh and rehydrated platelets. (a) Fresh platelets (30000x); (b) directly rehydrated platelets (30000x); (c) optimized rehydrated platelets $(20000 \times)$.
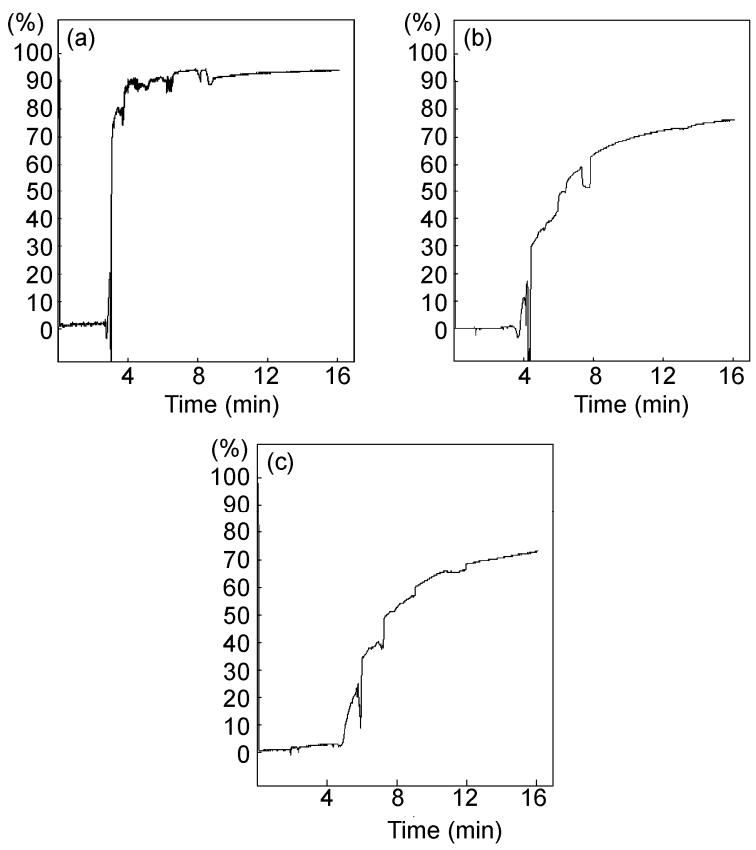

Figure 7 The aggregation traces of platelets. (a) Control (fresh platelets); (b) optimized rehydrated platelets; (c) directly rehydrated platelets.

aggregation percentage to thrombin $(1 \mathrm{U} / \mathrm{mL})$ for the freezedried platelets with the optimized rehydration was $82.8 \%$ of the value for fresh ones, and the maximum aggregation percentage to thrombin $(1 \mathrm{U} / \mathrm{mL})$ for the platelets with direct rehydration was $77.4 \%$ of the value for fresh ones. The response speed of the samples with optimized rehydration was much faster than that of the samples with direct rehydration, but slower than that of fresh platelets.

\section{Discussion}

Prehydration has been used in the rehydration of freeze- dried microorganisms [19]. Wolkers et al. [11] introduced this method in the freeze-drying of human platelets and obtained great success. They proposed that prehydration changes the state of the cell membrane and prevents membrane fusion and leakage of cytoplasmic components during the following rehydration process. Since prehydration itself was not the focus of their studies, only the prehydration ways for special cases were demonstrated. There remain a lot of problems to be solved.

The authors have carried out a primary study on the prehydration of $1 \mathrm{~mL}$ samples [17]. It was found that there was almost no difference between prehydration temperature $35^{\circ} \mathrm{C}$ and $37^{\circ} \mathrm{C}$. Among all prehydration durations tested, $15,30,60,90$ and $120 \mathrm{~min}$, the best result was obtained with the duration of $15 \mathrm{~min}$. In this paper, all prehydration was done at $37^{\circ} \mathrm{C}$. As the volume of the sample increased from 1 to $2 \mathrm{~mL}$, the optimal prehydration duration increased from 15 to $90 \mathrm{~min}$. This increment can be easily understood because the mass transfer resistance of water vapor increased with the thickness of the sample. The existence of optimal prehydration duration for $2 \mathrm{~mL}$ samples as well as for $1 \mathrm{~mL}$ samples further supposes that there should have a range for water content in which the prehydrated platelets survive the highest. If the water content is lower than a certain value, the cell membrane will not absorb enough water to avoid state transition during rehydration. If the water content is higher than a limit, free water appears and so does high concentration solution (HCS). HCS will bring damages to cells, as illustrated by many literatures [20]. From the present and the previous studies, the water content for optimized prehydration varied from $4.8 \%$ [17] to $6.1 \%$. It has to be noted that the water content is average value for the whole samples, in fact the distribution of water in the samples is not homogeneous. Knowing the distribution will help define more accurate range for water content, this needs more studies relevant to thermodynamic and kinetic properties. It may be reasonably inferred that for thicker sample prehydration, it will be more difficult to ensure appropriate water content for each part of the sample, therefore the cell recovery will be negatively impacted.

The importance of the rehydration solution has been addressed by several studies. For human platelets, the inclusion of platelet-poor plasma seems to be beneficial. Wolkers et al. [12] tested PBS: plasma: water (1:2:1) and plasma: water (3:1), while Fan et al. [17] tested 50\% plasma. The concentration of plasma will surely have impact due to the difference of osmolality. Here, special experiments were designed to study the effect of the concentration of plasma. Four concentrations of plasma, 25\%, 50\%, 75\% and $100 \%$, were investigated. According to the data of recovery rate and PDW, 75\% plasma may be the optimal rehydration solution. This result makes a progress compared with the authors' previous study.

After the optimization of the rehydration process, the recovery of rehydrated platelets has been improved. In the 
study of our group by Zhou et al. [14], the maximum aggregation (stimulated by $1 \mathrm{U} / \mathrm{mL}$ thrombin) of rehydrated platelets reached $52 \%$ in $5 \mathrm{~min}$. In this study, the aggregation (stimulated by $1 \mathrm{U} / \mathrm{mL}$ thrombin) of rehydrated platelets reached $63 \%$ in $4 \mathrm{~min}$, and the maximum aggregation reached $82.8 \%$. In the study of Walkers et al. [12], the maximum aggregation of rehydrated platelets reached $83.3 \%$ in $3 \mathrm{~min}$. The improvement of the aggregation speed needs more studies in the future.

\section{Conclusions}

The rehydration condition for lyophilized human platelets is optimized from the aspect of prehydration duration and rehydration solution. Among the four concentrations tested, $75 \%$ platelet-poor plasma performs best as rehydration solution. Among the six prehydration durations tested at $37^{\circ} \mathrm{C}$, $15 \mathrm{~min}$ is the most appropriate time for $1 \mathrm{~mL}$ sample and 90 $\min$ is the most appropriate time for $2 \mathrm{~mL}$ sample. It is supposed that to get the highest recovery of rehydrated platelets an optimal range of water content should be controlled when prehydration finishes. Further studies are needed to understand the physical process and offer more accurate knowledge.

This work was supported by the National Natural Science Foundation of China (50606032), Specialized Research Fund for the Doctoral Program of Higher Education (20070335145) and Scientific Research Foundation for Advanced Talents, Nanjing University of Aeronautics and Astronautics, China (1001-909382).

1 Kim S G, Chung C H, Kim Y K, et al. Use of particulate dentin-plaster of Paris combination with/without platelet-rich plasma in the treatment of bone defects around implants. Int J Oral Maxillofac Implants, 2002, 17: 86-94

2 Marx R E, Carlson E R, Eichstaedt R M, et al. Platelet-rich plasma: Growth factor enhancement for bone grafts. Oral Surg Oral Med Oral Pathol Oral Radiol Endod, 1998, 85: 638-646

3 Bhanot S, Alex J C. Current applications of platelet gels in facial plastic surgery. Facial Plastic Surg, 2002, 18: 27-33
4 Bock M, Schleuning M, Heim M U, et al. Cryopreservation of human platelets with dimethyl-sulfoxide: Changes in biochemistry and cell function. Transfusion, 1995, 35: 921-924

5 Bakaltcheva I, O'Sullivan A M, Hmel P, et al. Freeze-dried whole plasma: Evaluating sucrose, trehalose, sorbitol, mannitol and glycine as stabilizers. Thrombosis Res, 2007, 120: 105-116

6 Zhou X L, He H, Liu B L, et al. Effects of glycerol pretreatment on recovery and antioxidant enzyme activities of lyophilized red blood cells. Cryo Lett, 2008, 29: 285-292

7 Shuja F, Shults C, Duggan M, et al. Development and testing of freeze-dried plasma for the treatment of trauma-associated coagulopathy. J Trauma, 2008, 65: 975-985

8 Fleming J A, Hoekstra E J, Moniaga V, et al. Reuse prevention syringes for reconstitution of lyophilized vaccines: Operational study and UNICEF plans for expanding introduction. Int J Occup Environ Health, 2009, 15: 9-13

9 Zhou J, Liu J H. Progress of research on lyophilized preservation of Platelets (in Chinese). J Exp Hematol, 2004, 12: 542-545

10 Read M S, Reddick R L, Bode A P, et al. Preservation of hemostatic and structural properties of rehydrated lyophilized platelets: Potential for long-term storage of dried platelets for transfusion. Proc Natl Acad Sci USA, 1995, 92: 397-401

11 Wolkers W F, Walker N J, Tablin F, et al. Human platelets loaded with trehalose survive freeze-drying. Cryobiology, 2001, 42: 79-87

12 Wolkers W F, Walker N J, Tamari Y, et al. Towards a clinical application of freeze-dried human platelets. Cell Preserv Technol, 2003, 1: 175-188

13 Tang M K, Wolkers W F, Crowe J H, et al. Freeze-dried rehydrated human blood platelets regulate intracellular $\mathrm{pH}$. Transfusion, 2006, 46: $1029-1037$

14 Zhou X L, Zhu H, Zhang S Z, et al. Freeze-drying of human platelets: Influence of saccharide, freezing rate and cell concentration. Cryo Lett, 2007, 28: 187-96

15 Han Y, Quan G B, Liu X Z. Efect of reconstitution solutions on the recovery of lyophilized red blood cells (in Chinese). Chin J Blood Transfusion, 2004, 17: 10-12

16 Shu Z Q, Liu Z, Chen W W, et al. The rehydration injury study of freeze-dried red blood cells during rehydration. In: International Con-gress of Refrigeration, Beijing. 2007

17 Fan J L, Xu X G, Zhang S Z, et al. Experimental study on rehydration conditions of freeze-dried platelets. J Zhejiang Univ-SC A, 2009, 10: 697-703

18 Chen H. The assay sense of MPV, PCT and PDW of hemophiles' platelets (in Chinese). Appl J General Practice, 2006, 4: 479

19 Wolkers W F, Tablin F, Crowe J H. From anhydrobiosis to freeze- drying of eukaryotic cells. Comp Biochem Physiol A, 2002, 131: 535-543

20 Acker J P, Fowler A, Lauman B, Crowe J H. Survival of desiccated mammalian cells: Beneficial effects of isotonic media. Cell Preserv Technol, 2002, 1: 129-140

Open Access This article is distributed under the terms of the Creative Commons Attribution License which permits any use, distribution, and reproduction in any medium, provided the original author(s) and source are credited. 